\title{
28 Research Square \\ A S-Type Bistable Locally-Active Memristor and Its Application in Oscillator Circuit
}

Chunlai Li ( $\square$ lichunlai33@126.com )

Hunan Institute of Science and Technology

Haodong Li

Hunan Institute of Science and Technology

\section{Wenwu Xie}

Hunan Institute of Science and Technology

Jianrong Du

Hunan Institute of Science and Technology

\section{Research Article}

Keywords: Memristor, coexisting pinched hysteresis loops, non-volatile, local activity, nonlinear dynamics

Posted Date: May 17th, 2021

DOI: https://doi.org/10.21203/rs.3.rs-516128/v1

License: (a) This work is licensed under a Creative Commons Attribution 4.0 International License. Read Full License 


\section{Abstract}

In this paper, a S-type memristor with tangent nonlinearity is proposed. The introduced memristor can generate two kinds of stable pinched hysteresis loops with initial conditions from two flanks of the initial critical point. The power-off plot verifies that the memristor is nonvolatile, and the DC V-I plot shows that the memristor is locally active with the locally-active region symmetrical about the origin. The equivalent circuit of the memristor, derived by small-signal analysis method, is used to study the dynamics near the operating point in the locally-active region. Owing to the bistable and locally-active properties and S-type DC V-I curve, this memristor is called S-type BLAM for short. Then, a new Wien-bridge oscillator circuit is designed by substituting one of its resistances with S-type BLAM. It find that the circuit system can produce chaotic oscillation and complex dynamic behavior, which is further confirmed by analog circuit experiment.

\section{Full Text}

Due to technical limitations, full-text HTML conversion of this manuscript could not be completed. However, the manuscript can be downloaded and accessed as a PDF.

\section{Figures}



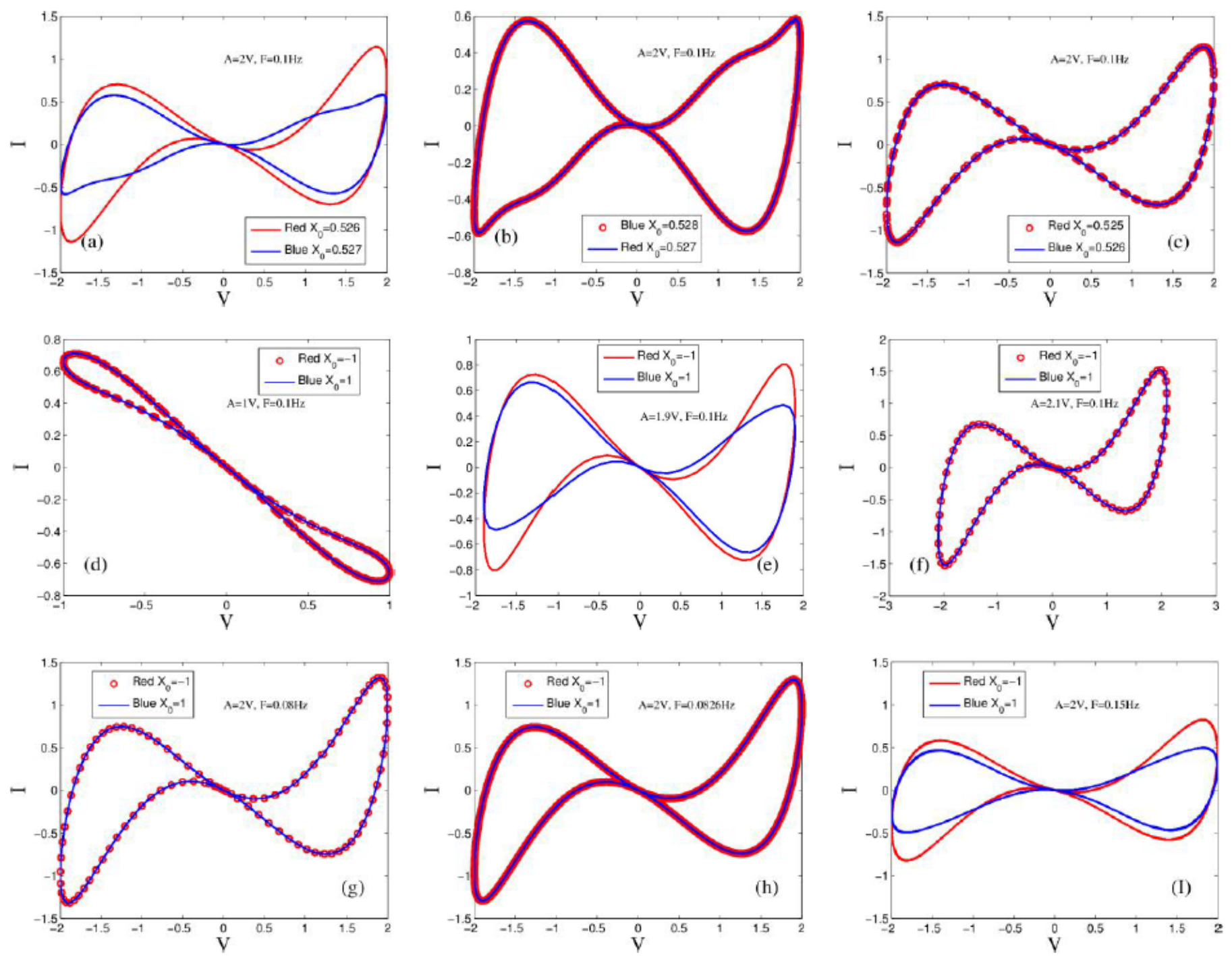

Figure 1

Coexisting pinched hysteresis loops with: different initial values of $(a) x(0)=0.526$ and $x(0)=0.527$, (b) $x(0)=0.527$ and $x(0)=0.528$; (c) $x(0)=0.525$ and $x(0)=0.526$; different amplitudes of (d) $A=1 \mathrm{~V},(e) A=1.9 \mathrm{~V}$, (f) $A=2.1 \mathrm{~V}$; different frequencies of (g) $F=0.08 \mathrm{~Hz}$, (h) $F=0.0826 \mathrm{~Hz}$, (I) $F=0.15 \mathrm{~Hz}$. 


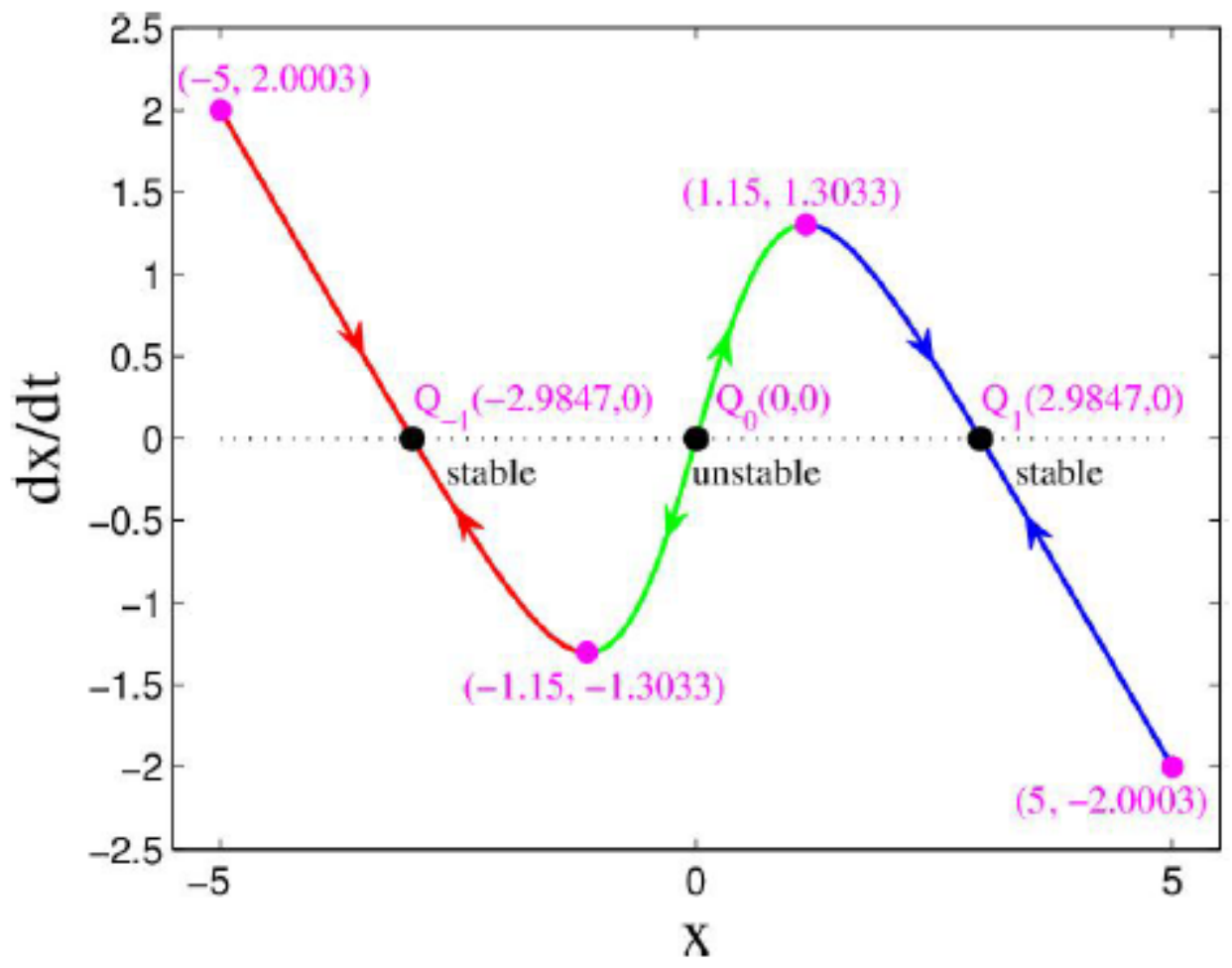

Figure 2

POP of S-type BLAM. 

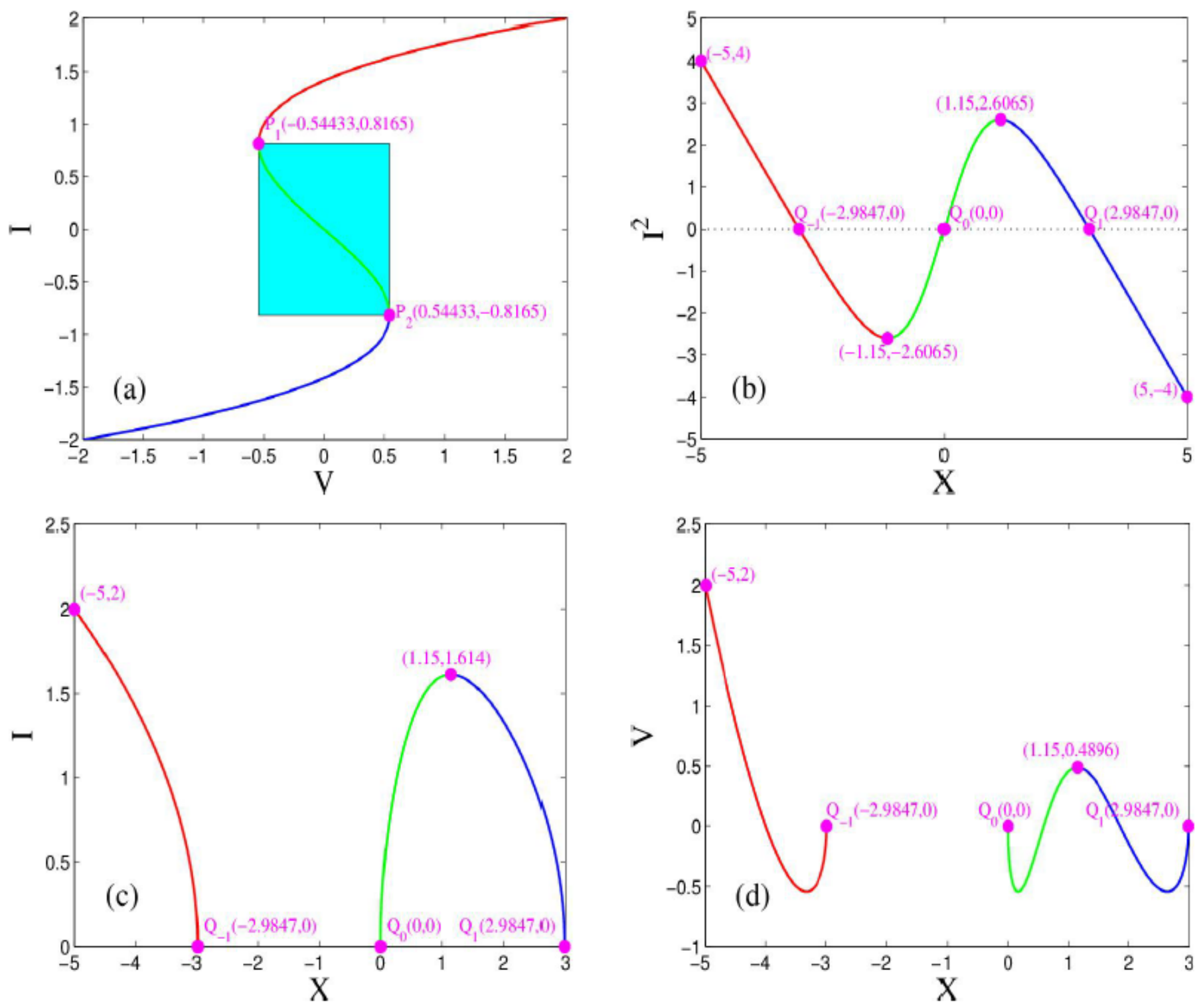

Figure 3

(a) DC V-I plot of S-type BLAM and equilibrium state curve on (b) X-I2 plane, (c) X-I plane and (d) X-V plane. 


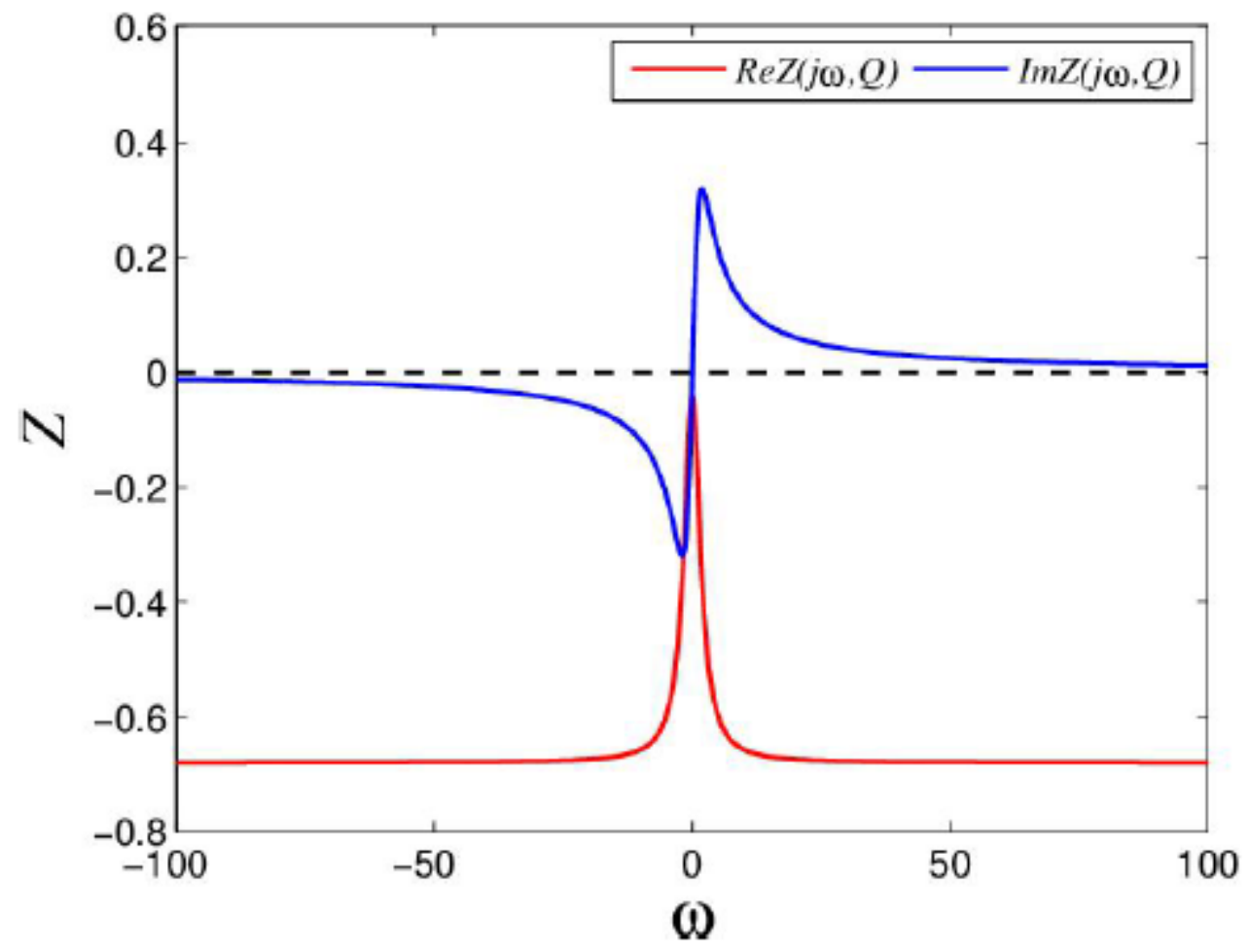

Figure 4

Frequency responses of S-type BLAM with $\mathrm{I}=0.8 \mathrm{~mA}$
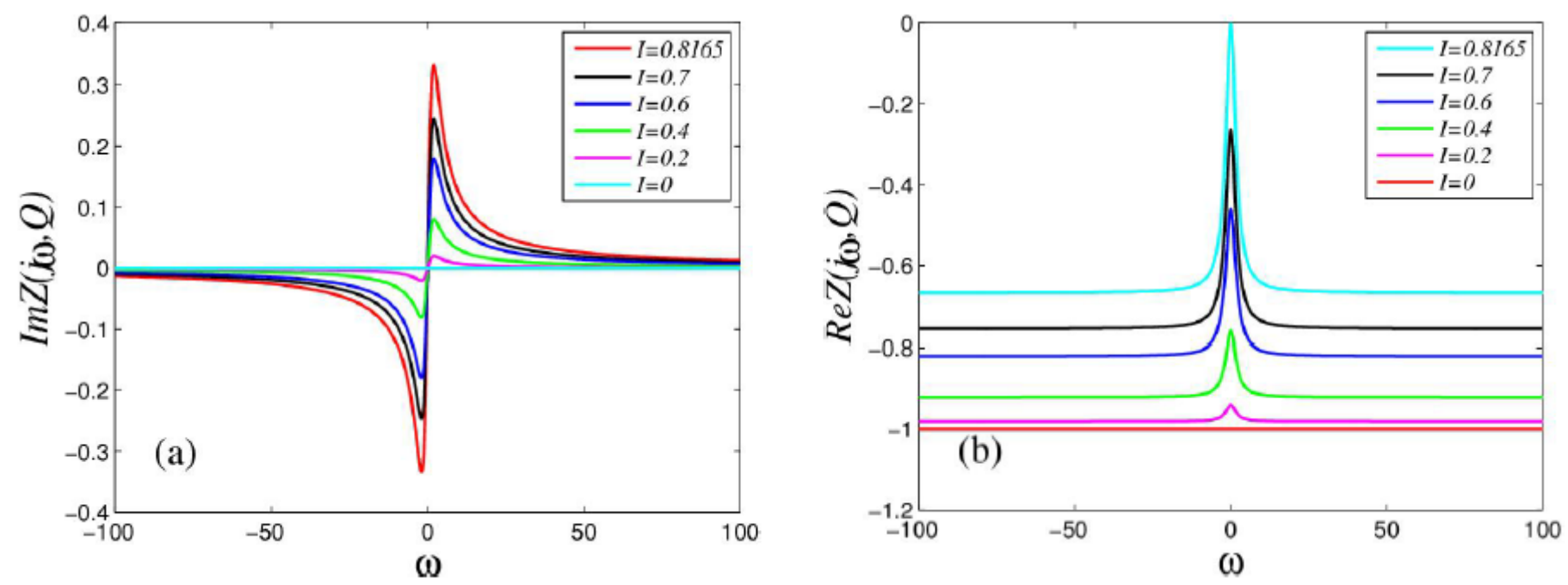

Figure 5

(a) Frequency responses of $\operatorname{ImZ}(j \omega, Q)$ about different $D C$ currents; (b) Frequency responses of $\operatorname{Re} Z($ $j \omega, Q)$ about different $D C$ currents 


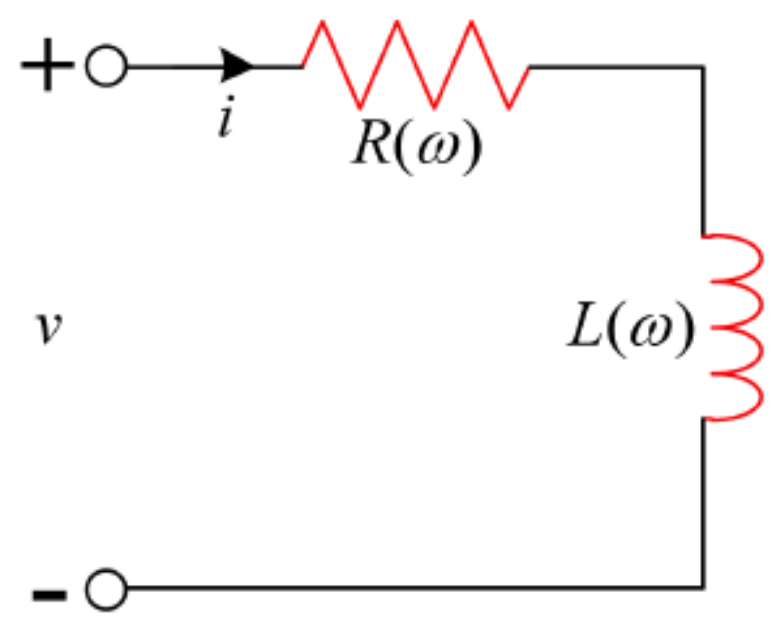

Figure 6

Small-signal equivalent circuit of the proposed memristor.

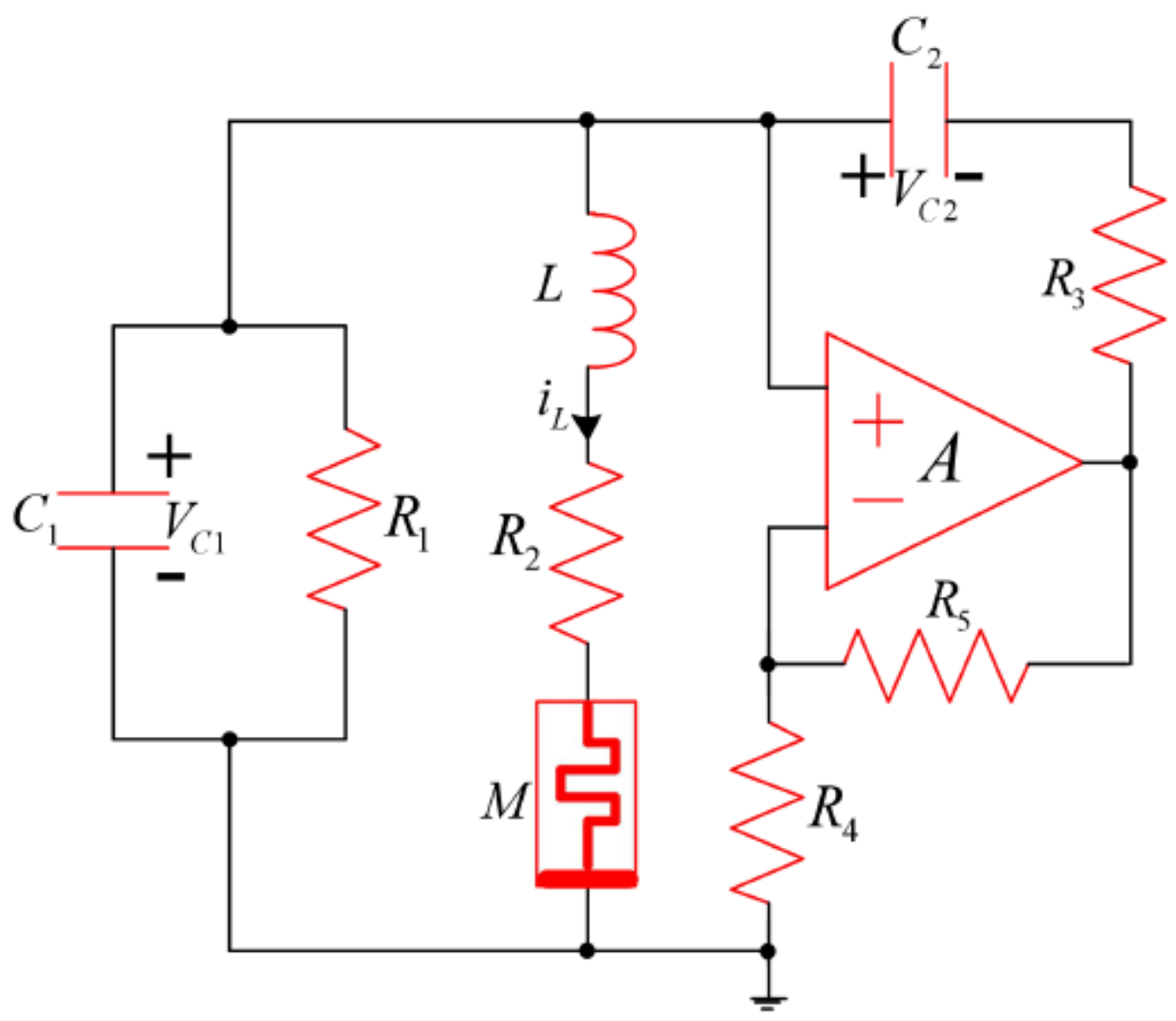

Figure 7

S-type BLAM-based Wien-bridge oscillator circuit. 


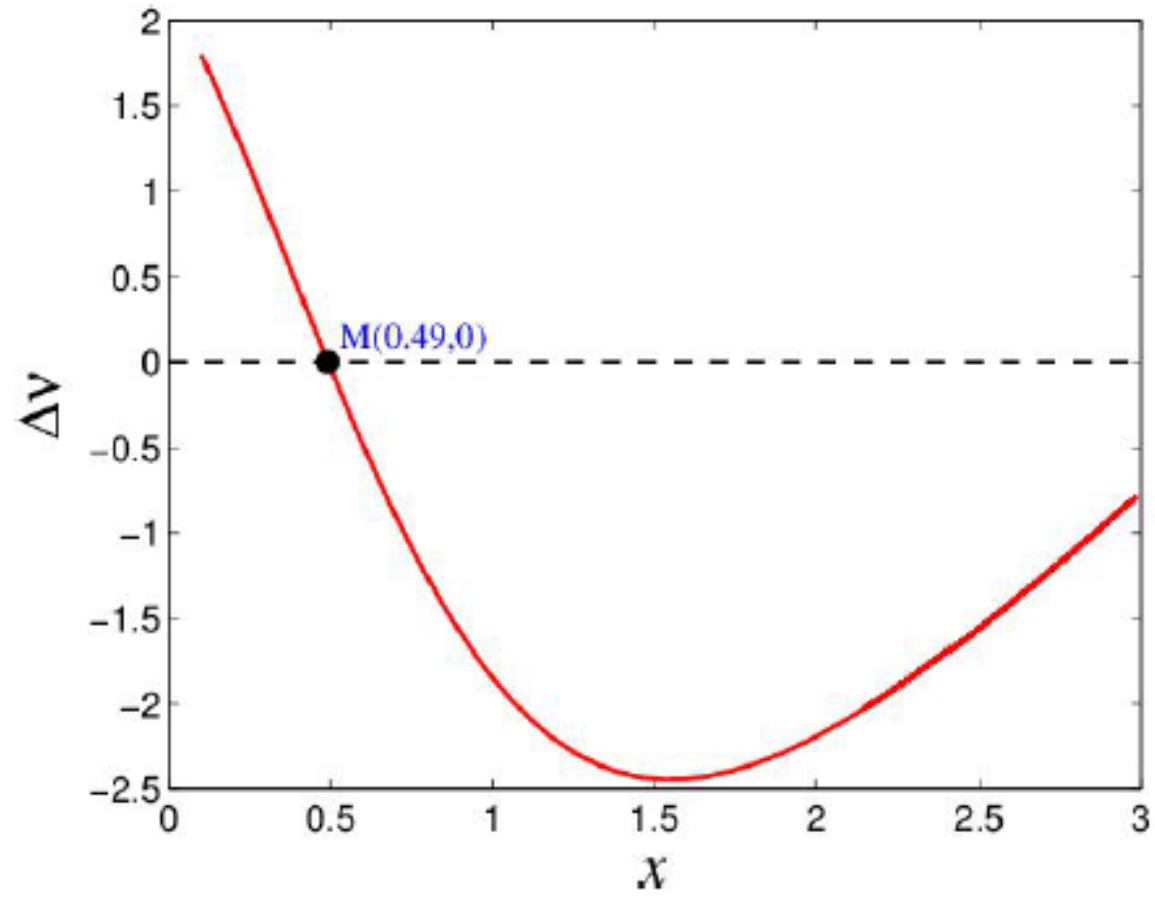

Figure 8

The plot of $\Delta v$ on the $\mathrm{x}$ system orbit.
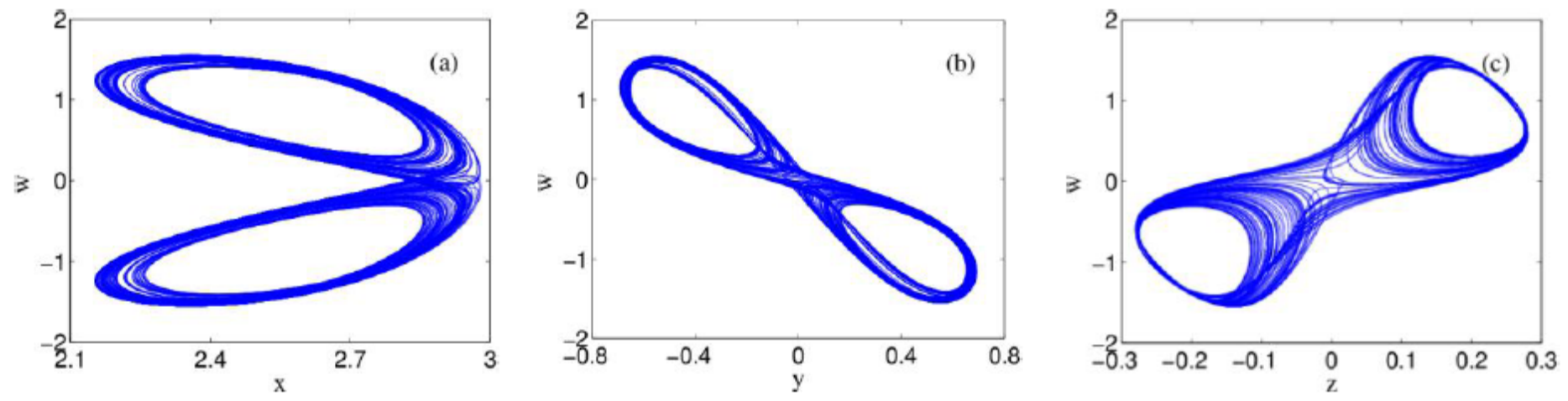

Figure 9

The phase diagram of system (20) in (a) x-w plane; (b) y-w plane. (c) z-w plane 

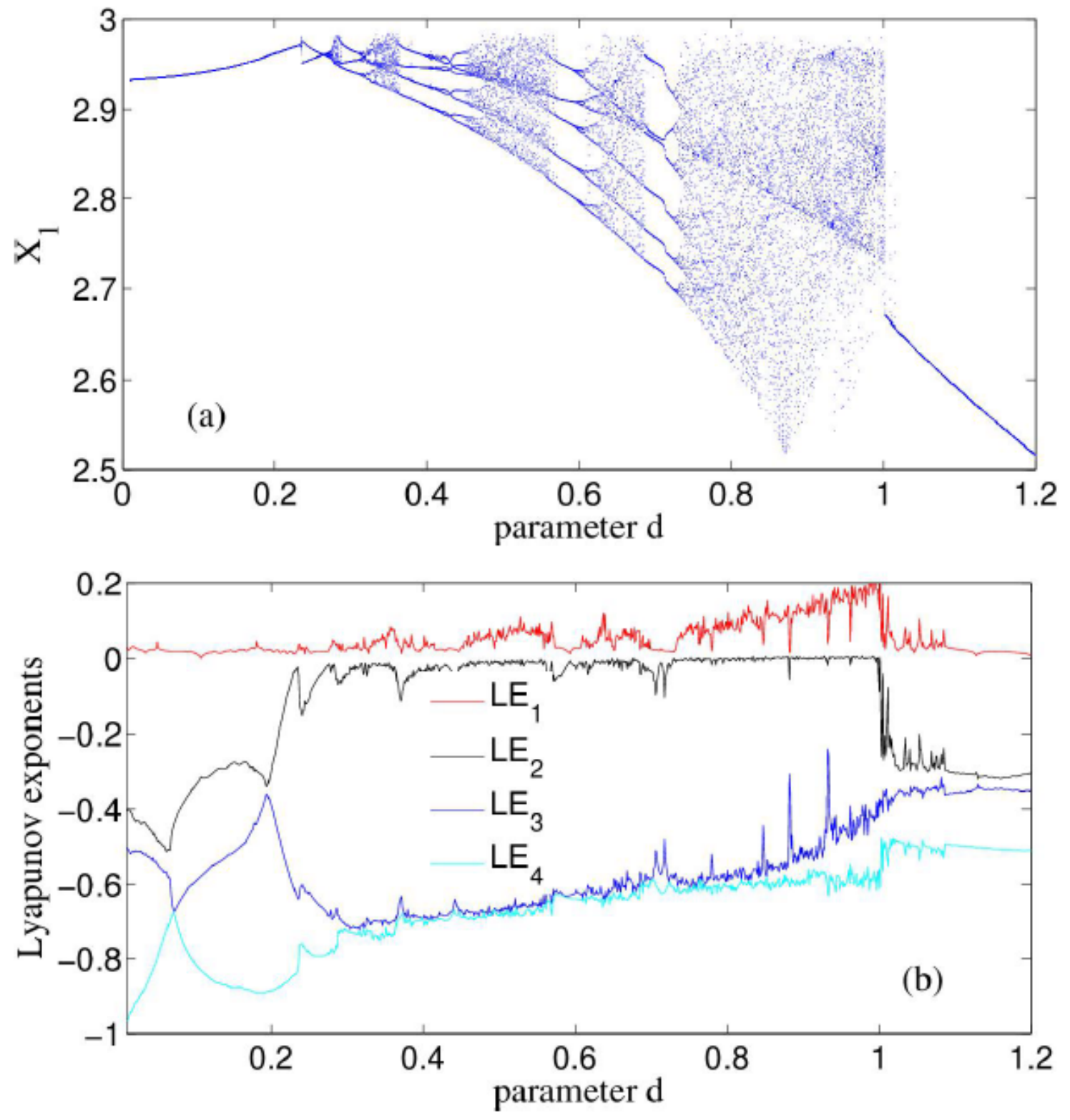

Figure 10

(a) Bifurcation diagram and (b) Lyapunov exponent spectrum versus parameter d 

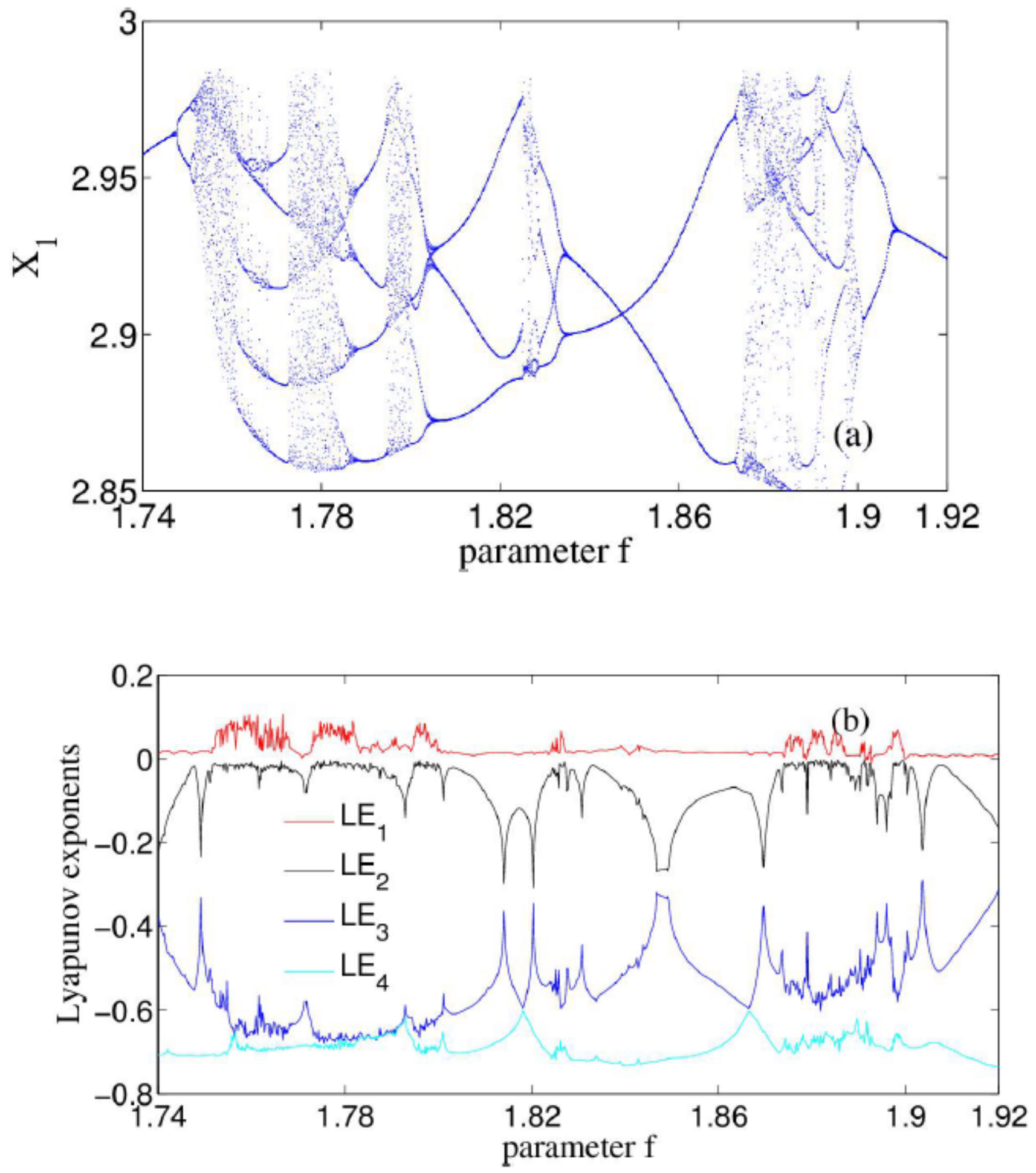

Figure 11

(a) Bifurcation diagram and (b) Lyapunov exponent spectrum versus parameter $f$ 

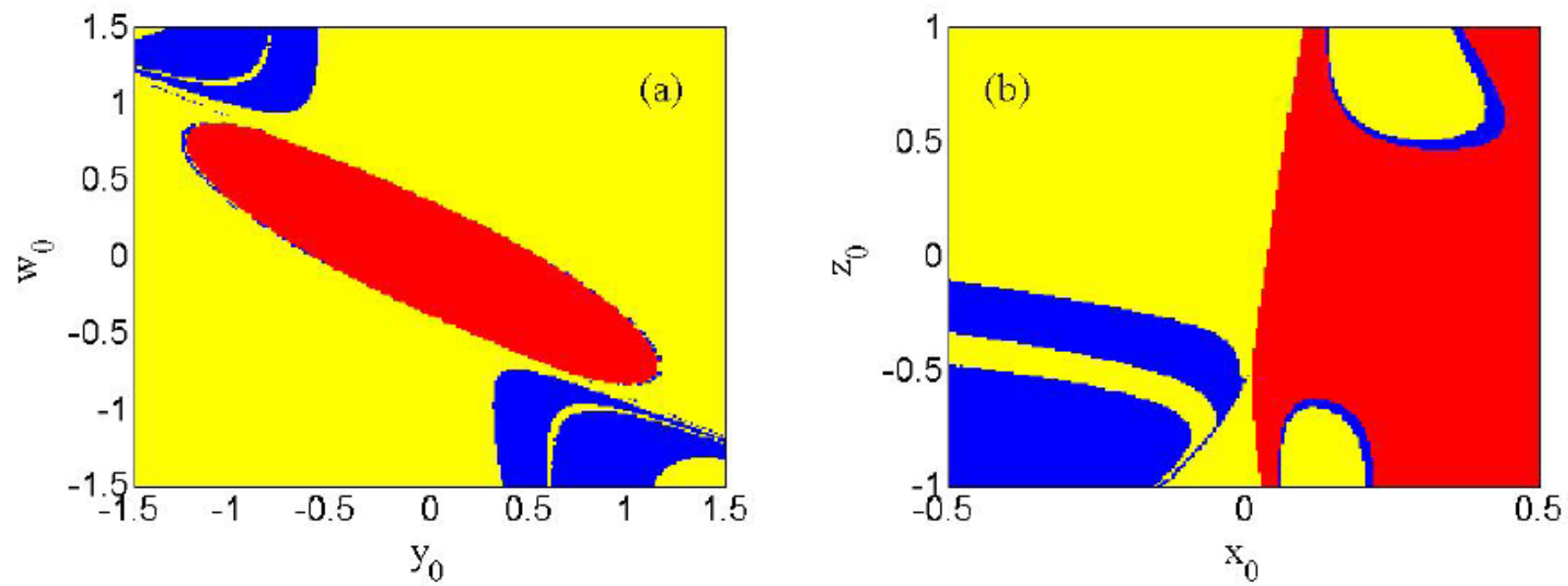

Figure 12

Local basins of attraction in (a) y0-w0 plane with $\mathrm{x} 0=0.1$ and $\mathrm{z} 0=0.1$; (b) $\mathrm{x} 0-\mathrm{z} 0$ plane with $\mathrm{y} 0=0.1$ and $\mathrm{w} 0=0.1$.
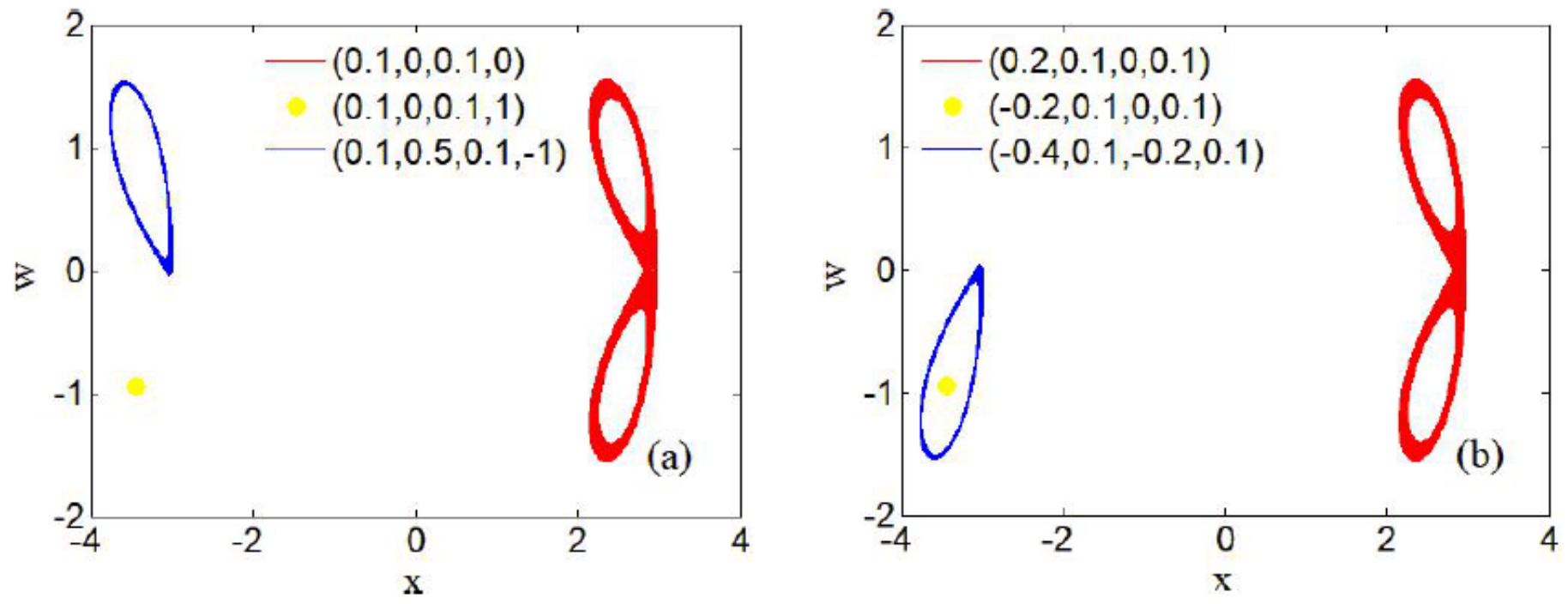

Figure 13

Different types of coexisting attractors in (a) different attraction regions of Fig.12 (a); (b) different attraction regions of Fig.12 (b). 

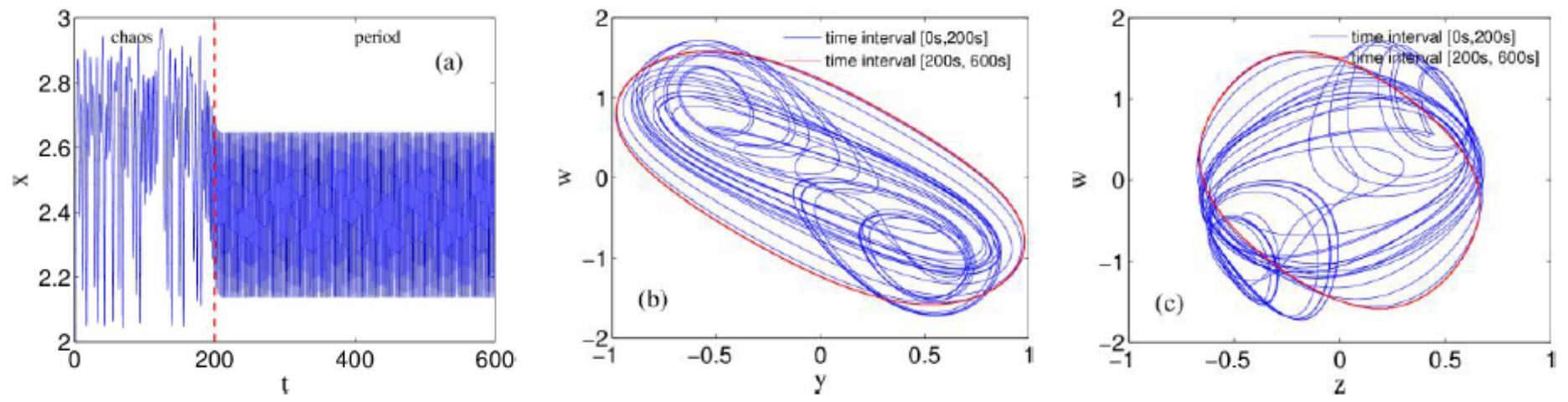

Figure 14

(a) Time-domain diagram of $x$ in the region of [0s, 600s]; phase portrait for the time intervals [0s, 200s] and [200s, 600s] in (b) y-w plane and (c) z-w plane.



Figure 15

Circuit diagram of memristor-based Wien-bridge circuit. 

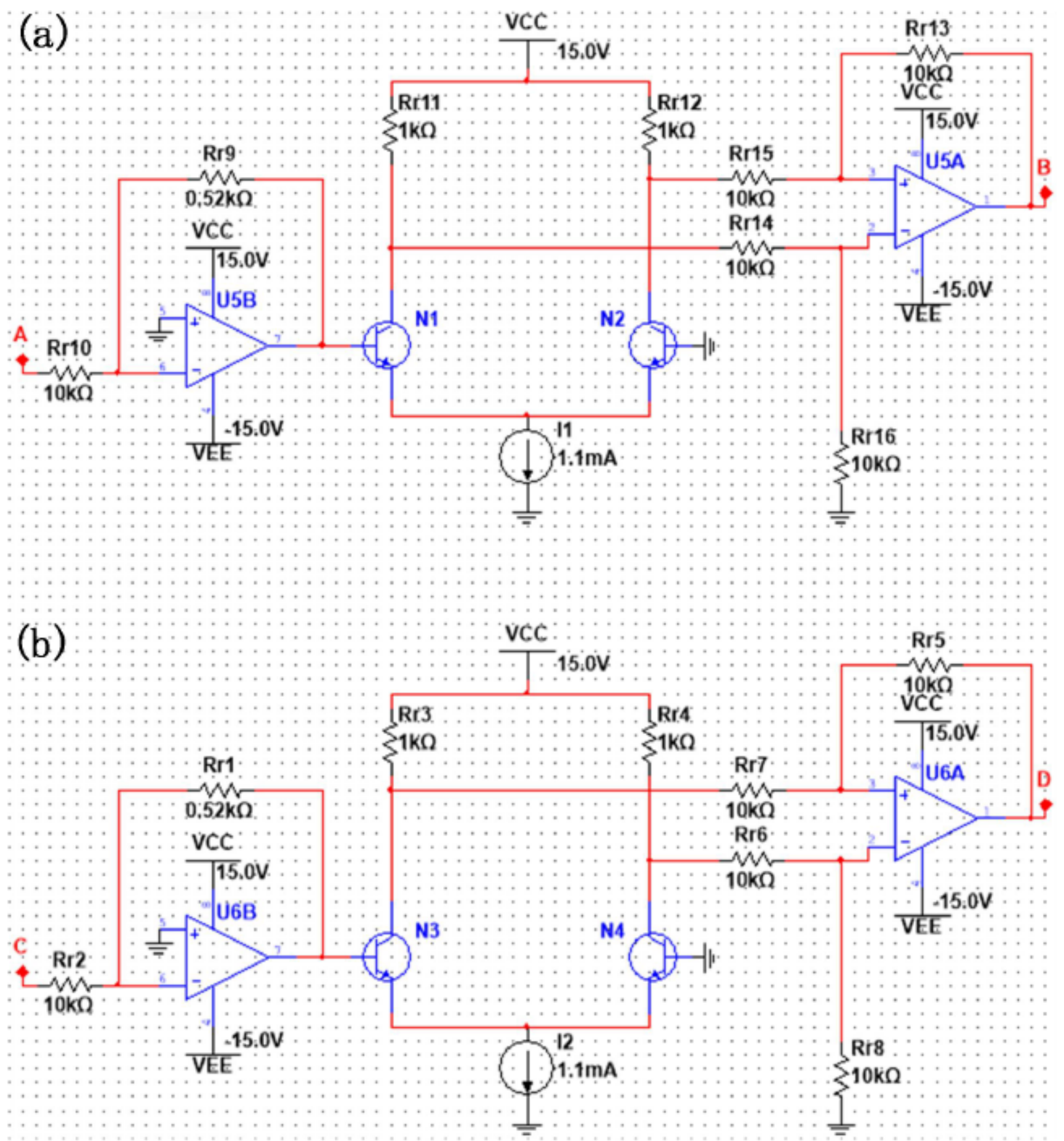

Figure 16

Circuit diagrams of (a) function $\tanh (\cdot)$ and (b) function $-\tanh (\cdot)$. 


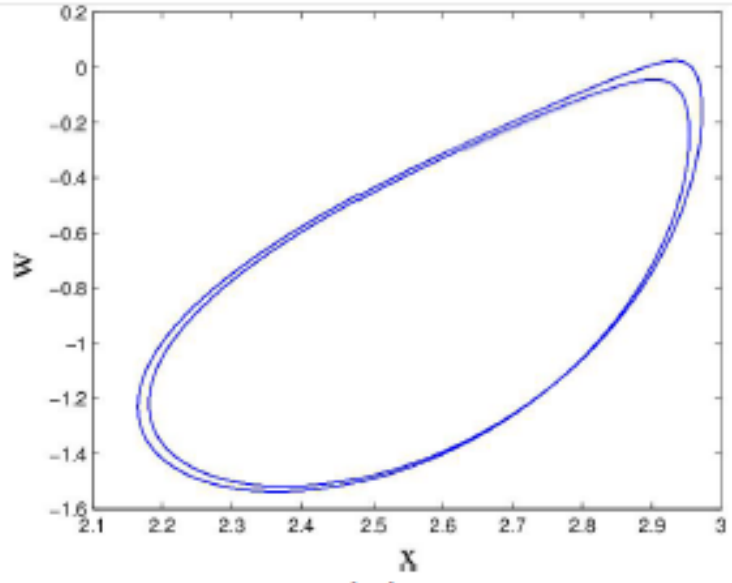

$\left(\mathrm{a}_{1}\right)$

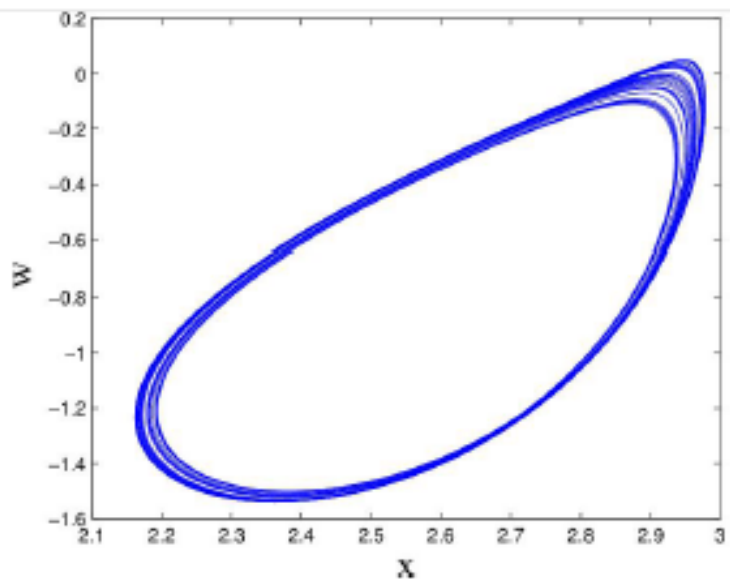

$\left(\mathrm{b}_{1}\right)$

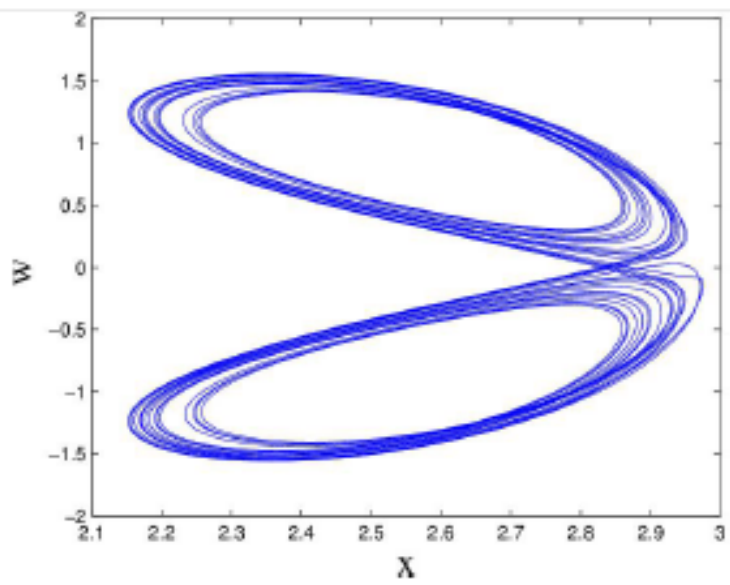

$\left(c_{1}\right)$

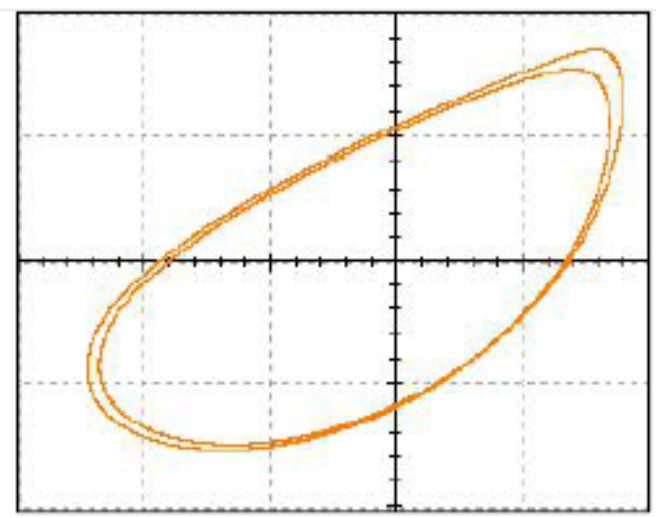

(a2)

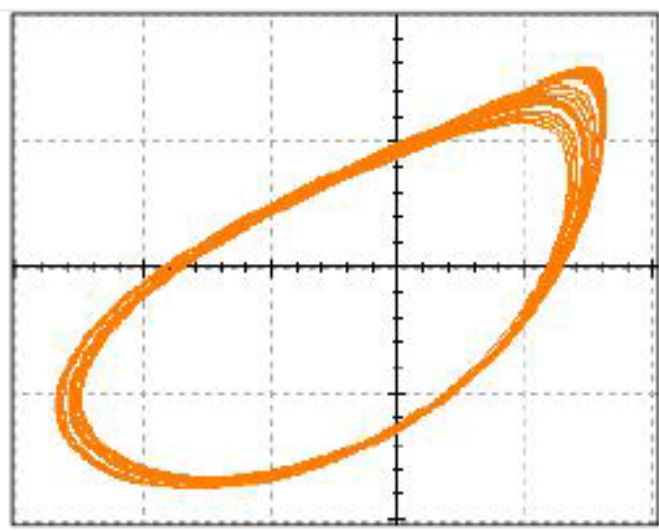

$\left(b_{2}\right)$

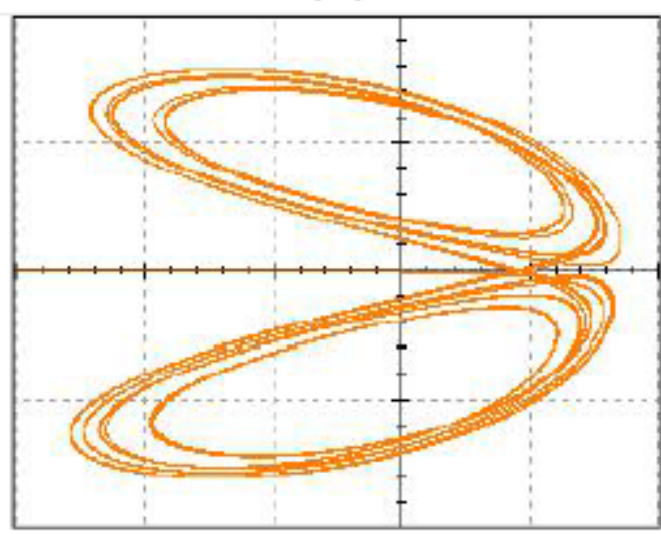

$\left(c_{2}\right)$

\section{Figure 17}

Numerical and experimental results for (a1-a2) period-2 attractor; (b1-b2) single scroll chaotic attractor and (c1-c2) double scroll chaotic attractor 